\title{
La traducción como arma propagandística: censura de Orwell, Abellio y Koestler en la España franquista
}

\author{
Purificación Meseguer \\ Universidad de Murcia \\ purificacion.meseguer@um.es
}

\begin{abstract}
Resumen: Este artículo trata de desentrañar el papel que desempeñó la traducción al servicio del franquismo. Para ello, se realiza un estudio de tres obras caracterizadas por un fuerte componente político y publicadas durante la dictadura: La escritura invisible, de Koestler; 1984, de Orwell; y Los ojos de Ezequiel están abiertos, de Abellio. Empleando una metodología mixta que combina el análisis cuantitativo y cualitativo de los datos, se pretende detectar ejemplos de manipulación y establecer patrones de comportamiento censor. Los resultados muestran una clara tendencia a la "metacensura", una estrategia que no busca suprimir contenido sino crear discursos favorables a los intereses franquistas, utilizando la traducción como arma propagandística.
\end{abstract}

Palabras clave: traducción; franquismo; censura; estrategias; propaganda.

\begin{abstract}
This paper aims to explore the role of translation under Franco's dictatorship. The study carried out compares three novels sharing a significant political component: The Invisible Writing, by Koestler; Nineteen Eighty Four, by Orwell; and Les Yeux d'Ezéchiel sont ouverts, by Abellio. The object is to identify examples of censorship manipulation and establish translational patterns by analysing -both quantitatively and qualitatively- the strategies detected in the translated texts. The analysis reveals a peculiar type of censorship, here coined as "metacensorship", which goes far beyond the traditional methods and does not limit itself to cut content, but rather to use and shape it with propaganda purposes.
\end{abstract}

Keywords: translation; Francoism; censorship; strategies; propaganda. 



\section{Introducción}

La censura franquista ha sido, a lo largo de los últimos años, objeto de estudio para los investigadores que han querido medir su impacto en la traducción y la recepción de textos vertidos al castellano en aquella época (Laprade, 2006; Lázaro, 2004; Merino, 2007; Rabadán, 2000; Santoyo, 2000). La mayoría de estos estudios explora el funcionamiento y las consecuencias de la censura, encargada a la sazón de regular la publicación de libros. Pero pese a que los manuscritos extranjeros eran vistos como una amenaza, fueron muchos los textos traducidos que vinieron a llenar el vacío cultural que habían dejado los miles de autores que, durante los primeros años de la dictadura, huyeron a Europa y Latinoamérica. A la luz de este hecho cabe pues preguntarse si la traducción, más que una amenaza, no representó en algún momento una oportunidad para configurar el sistema literario franquista. No se trata de medir aquí las consecuencias silenciosas del aparato censor, sino de indagar en el papel de la traducción al servicio del régimen. Así pues, este trabajo pretende ahondar en esta faceta de la traducción como herramienta propagandística, un fenómeno que ya ha sido estudiado en otros medios, como el audiovisual (Ruiz, 2005). Para alcanzar este objetivo, este artículo se centra en el estudio de tres obras que presen$\tan$ un fuerte componente político contrario a los intereses franquistas -1984, de George Orwell (1952); Los ojos de Ezequiel están abiertos, de Raymond Abellio (1955); y La escritura invisible, de Arthur Koestler (1974)- en cuyas traducciones se identificarán no solo ejemplos de manipulación textual sino también patrones de intervención censoria. Este trabajo se articula en torno a tres apartados. En primer lugar, se exploran aspectos contextuales relevantes del franquismo como los mecanismos de reacción puestos en marcha a nivel legal e institucional para neutralizar cualquier manera de pensar disidente que intentara filtrarse por medio de la literatura extranjera. En segundo lugar, se recogen los datos del análisis de las obras, que permite detectar cada intervención censoria. El análisis cuantitativo y cualitativo de las estrategias identificadas se complementa con el material extratextual que proporcionan los expedientes del Archivo General de la Administración (AGA) -archivo

\footnotetext{
* La presente investigación ha contado con financiación del Ministerio de Economía y Competitividad (FFI2013-45553-C3-3-P).
} 
que recoge el casi medio millón de expedientes de censura de libros generados en España desde 1939 hasta 1983- y las entrevistas que realizamos en 2013 y en el marco de esta investigación a Edward Douglas Laprade, pionero en el estudio de la censura en la recepción de obras extranjeras, Beatriz de Moura, a la sazón editora de Tusquets y Manuel Serrat Crespo, traductor en la época franquista, lo que nos permitirá desentrañar los motivos que impulsaron la censura de estas obras. Por último, se discuten los resultados obtenidos en las tres obras en su conjunto, donde se observa una tendencia, no a eliminar contenido, sino a reescribir con fines propagandísticos.

\section{La censura de libros bajo el régimen franquista}

El franquismo se instauró a base de medidas represivas, orientadas a acallar voces disidentes y a imponer una ideología autoritaria y católica. Durante los primeros años de la dictadura, se llevó a cabo una especie de purga intelectual, con el encarcelamiento de autores y editores, el cierre de editoriales y la destrucción de manuscritos (Ruiz Bautista, 2008). La aniquilación de la élite intelectual española y el aplastamiento del sector editorial llevaron a un empobrecimiento cultural generalizado, a un estado de adormecimiento que allanaría el camino a la entrada e instauración de los valores de la nueva España. La censura, un mecanismo eficaz de control de las publicaciones, se instauró con un doble propósito: el de mantener y fortalecer los valores que pregonaba el régimen, y el de filtrar todo material "contaminante" procedente del exterior. Aunque existen ejemplos de manipulación previos, la censura no se institucionalizó hasta 1938, con la entrada en vigor de la Primera Ley de Prensa, inspirada en los modelos de propaganda de la Italia fascista y los diseñados por Goebbles en la Alemania nazi (Cisquella et alii, 1977: 19). Cualquier obra se veía sometida a un riguroso examen con el que se pretendía borrar o modificar el contenido susceptible de herir las sensibilidades de la sociedad española. Aparte de las listas de autores "malditos", se establecieron criterios censorios que, aunque tácitos, se examinaban: se censuraba cualquier ataque a la Iglesia y la religión, las críticas al régimen, a Franco y al ejército, sin olvidar todo aquello que pudiera considerarse indecoroso (Cisquella et alii, 1977; Abellán, 1980). Si bien se ha elucubrado mucho sobre la arbitrariedad de este sistema -en parte, por las inconsistencias halladas en el análi- 
sis de los expedientes que han desbrozado muchos de los autores que han abordado este fenómeno-, lo cierto es que los censores obedecían estos criterios, que fueron regulados y acatados a partir de las numerosas leyes y normas aprobadas, como la "censura previa", por la que todos los editores estaban obligados a presentar un ejemplar de la obra que quisieran publicar y que quedaba sujeto a las tachaduras que el censor considerase oportunas. Precisamente para dotar de sistematicidad al aparato censor, se aprobó en 1966 la Ley de Prensa e Imprenta. Aunque en principio se vio en ella la promesa de una nueva etapa de libertad, lo cierto es que su aprobación trajo consigo una serie de medidas coercitivas que abrieron una etapa de adoctrinamiento (Savater, 1996). El sistema quedó efectivamente reforzado gracias, en parte, al sector editorial que, sometido a presiones, acabó participando de esta represión cultural e intelectual. La derogación de la "censura previa" trajo la implantación de la "consulta voluntaria", aparentemente más flexible: los editores ya no estaban obligados a presentar a priori sus proyectos editoriales a censura. Las consecuencias de publicar un texto equivocado, sin embargo, podían ir desde el secuestro de una edición hasta cuantiosas multas. Según Abellán (1982: 173):

Esta ley obligó a que los editores fueran más precavidos que antes y censuraran previamente manuscritos o galeradas so pena de ser considerados cómplices de los delitos en los que la obra publicada pudiera todavía incurrir.

Que el editor presentara su proyecto a consulta tampoco le libraba de esta suerte: su proyecto podía recibir el "silencio administrativo", una postura a la que se acogía la Administración en situaciones puntuales. Según Abellán (1980: 149-150), se trataba de una solución inhibitoria, un modo de devengar la responsabilidad en el editor. De este modo, el sistema censor se aseguraba de que todos los agentes involucrados en un proyecto editorial participaran de un modo u otro en la construcción del sistema literario franquista, no solo vetando a los escritores que pudieran suponer una amenaza contra el régimen sino favoreciendo a aquellos que pudieran proporcionar material favorable a la causa. Pero también se publicaron autores polémicos; en el caso de Green o Zola, la censura transformó el discurso original para crear discursos favorables al régimen (Meseguer, 2015). Las obras que se analizan aquí son un 
ejemplo más de cómo la traducción se convirtió en una poderosa herramienta de propaganda.

\section{Análisis de las obras de temática política}

El corpus objeto de estudio está compuesto por tres obras escritas originariamente en inglés y francés (Nineteen Eighty Four, de George Orwell, 1949; Les Yeux d'Ézéchiel sont ouverts, de Raymond Abellio, 1949; y The Invisible Writing, de Raymond Abellio, 1954) y por sus traducciones al español, publicadas en la dictadura franquista (1984, de George Orwell, 1952; Los ojos de Ezequiel están abiertos, de Raymond Abellio, 1955; y La escritura invisible, de Arthur Koestler, 1974). Se trata de un conjunto heterogéneo, formado por tres obras de características variopintas en cuanto a autores, lenguas de origen y fechas de publicación, sin mencionar el mundo literario que las distingue, a nivel de trama, contextualización o estilo. El denominador común que comparten: presentar un destacado contenido ideológico disidente y haber sido filtradas por la Administración franquista previamente a su publicación. Como paso previo al análisis de las tres obras en su conjunto, donde se perfilarán patrones de comportamiento censor, en este apartado se presenta el análisis individual de las obras. Así, se da un breve repaso al examen al que fue sometida la obra antes de su publicación y se detallan los resultados del análisis textual realizado, en el que se identifican cuatro estrategias: supresión (contenido censurable en el TO que pasa a desaparecer en el TM), sustitución (contenido censurable en el TO que, a partir de una sustitución, queda neutralizado en el TM), ampliación (adición de contenido que no aparece en el TO y con el que se pretende transmitir un mensaje ideológico en el TM) y reescritura (tipo de sustitución que se sirve del contenido censurable que presenta el TO para introducir un cambio ideológico en el TM).

\subsection{4, de George Orwell}

1984 llegó a los lectores españoles a través de la traducción de Rafael Vázquez Zamora y en una edición de 312 páginas. La editorial Destino, que la publicaría en 1952, presentó la novela a consulta el 17 de julio de 1950, un proceso administrativo que recoge el expediente 43632/50 del AGA. El primer censor emitió un informe días más tarde donde subraya 
las posibilidades de esta obra, interesante a su juicio, "ya que su tendencia es anticomunista". No puede, sin embargo, autorizar su publicación puesto que presenta numerosos pasajes que podrían resultar comprometedores y deberían matizarse. Destino presentó más tarde una segunda versión, alegando que existen razones de peso para autorizar su publicación, no solo porque "está prohibida en todos los países de influencia soviética, siendo muy grande su aceptación en Europa y en América", sino, sobre todo, porque "constituye un formidable alegato contra el régimen comunista". Pero para alcanzar este fin, la novela debía someterse a una remodelación; 1984 no solo sufrió las tachaduras propuestas por la Administración, sino que fue objeto de numerosas alteraciones. Todos entraron en juego para transformar la novela: traductor y editor, en un ejercicio de censura interna; y censores, que se adhirieron a los criterios de la censura institucional. Esta nueva creación vendría a favorecer los intereses del régimen con un nuevo mensaje: alertar no de los peligros del totalitarismo en general sino del comunismo en particular. El análisis realizado refleja 53 ejemplos de intervención censoria: 42 supresiones, 8 reescrituras, 2 sustituciones y 1 ampliación.

\begin{tabular}{|c|c|c|}
\hline Estrategia & Ejemplos & Porcentaje \\
\hline Supresión & 42 & $79 \%$ \\
\hline Reescritura & 8 & $15 \%$ \\
\hline Sustitución & 2 & $4 \%$ \\
\hline Ampliación & 1 & $2 \%$ \\
\hline
\end{tabular}

Tabla 1. Cuantificación de estrategias censorias halladas en 1984

La tabla 1 revela que la supresión fue la estrategia predominante con casi un $80 \%$ de los casos. El ejemplo más significativo ha de encontrarse en el apéndice de la propia novela de Orwell, titulado "Los principios de la neolengua", un total de once páginas del TO. Este apéndice, que describe y explica el origen de la neolengua y su función dentro de este Estado totalitario ideado por Orwell, contiene toda una serie de referencias políticas que lo convierten en un discurso ideológico políticamente incorrecto. Aquí radica la verdadera esencia de 1984, ya que Orwell pone de manifiesto una crítica a los sistemas totalitarios, y no solo al comunista. De esta manera, se encuentran menciones explícitas al socialismo, al fascismo, al marxismo y al nazismo. La censura no se limi- 
ta aquí a un ejercicio de maquillaje; amputa limpiamente un miembro de la obra, pasajes, como este, que amenazaban con frustrar el objetivo de los censores:

[...] it had been noticed that the tendency to use abbreviations of this kind was most marked in totalitarian countries and totalitarian organizations. Examples were such words as nazi, Gestapo, Comintern, Inprecorr, Agitprop [...] in Newspeak it was used with a conscious purpose. It was perceived that in thus abbreviating a name one narrowed and subtly altered its meaning, by cutting out most of the associations that would otherwise cling to it. The words communist international, for instance, call up a composite picture of universal human brotherhood, red flags, barricades, Karl Marx, and the Paris Commune. The word Comintern, on the other hand, suggests merely a tightly-knit organization and a well-defined body of doctrine [...] Comintern is a word that can be uttered almost without taking thought, whereas communist international is a phrase over which one is obliged to linger at least momentarily (Orwell, 1949: 277).

Pero la supresión no fue la única estrategia detectada. La traducción actuó como una poderosa herramienta de remodelación, valiéndose también de la reescritura, la sustitución o la ampliación. Como veremos a continuación, el mismo ejercicio de remodelación ideológica tuvo lugar en Los ojos de Ezequiel están abiertos y La escritura invisible. Al igual que la censura favoreció la publicación de autores amables con el régimen, tal y como apunta Laprade en su entrevista, cabe preguntarse si la censura no permitió también la entrada de autores menos amables, pero cuyos discursos encerraran una doble lectura y pudieran resultar convenientes ${ }^{2}$. Lázaro (2002: 5) ha sabido discernir las diferentes lecturas que tiene la novela que nos ocupa, haciendo hincapié en el hecho de que la lectura que se ofrecía bajo el franquismo no era la pretendida por su autor, idea que sostuvo apoyándose en testimonios del propio Orwell:

Son los años de la Guerra Fría y 1984 está siendo acogida como una denuncia del antiindividualismo soviético, una advertencia contra el "peligro rojo". Esta interpretación es sesgada e incompleta, puesto que

\footnotetext{
${ }^{2}$ Entrevista realizada a Edward Dougla Laprade en el marco de la presente investigación.
} 
de nuevo Orwell tiene en mente las tendencias totalitarias en general, ya sean de izquierdas o de derechas.

Y de este modo, 1984, una novela que pretende advertir de las derivas totalitarias, acabó acondicionada para servir los intereses contra los cuales iba precisamente dirigida.

\subsection{Los ojos de Ezequiel están abiertos, de Raymond Abellio}

Los ojos de Ezequiel están abiertos llegó a España en 1955 en una edición de 410 páginas. La publicó Escelicer, con traducción de José Vila Selma. La editorial presentó la novela a consulta el 7 de septiembre de 1955, tal y como recoge el expediente 4586/55, de 19 páginas, el más voluminoso de las tres novelas objeto de estudio. Y es que Escelicer no recibiría la aprobación hasta semanas más tarde, después de que la obra hubiera sido objeto de una remodelación en el que tomaron parte varios censores. El primer informe del expediente advierte ya de los peligros de esta novela de la que son protagonistas "unos finos ejemplares de las Brigadas Internacionales", que atentaría contra el ambiente español desde un "punto de vista ideológico, moral y político". El censor considera que esta "arenga anarquista" es peligrosa y, por lo tanto, desaconseja su publicación. Sin embargo, hace una observación muy interesante: la moraleja que puede desprenderse sería "positiva, por una minoría muy reducida", si no fuese por lo corrosivo de sus diálogos, y marca las páginas que profieren ataques tanto contra la moral como contra la Iglesia y el régimen, un total de una treintena. La resolución recogida en el siguiente informe, que aparece dos semanas más tarde, es favorable pero el censor de esta nueva lectura ratifica únicamente la eliminación de cuatro pasajes de criterio religioso. En cuanto al resto, solicita que sean evaluadas por otro censor con criterio político. No existe, sin embargo, otro documento que nos dé a conocer ningún dato sobre la nueva valoración; a continuación, no aparece más que un documento en el que el propio editor afirma que la novela ya ha sido "convenientemente 'modelada' por Vila Selma". A la luz de este comentario, cabe conjeturar que la censura interna contribuyó también a la remodelación de Los ojos de Ezequiel están abiertos. El análisis realizado revela 184 ejemplos de intervención censoria: 66 reescrituras, 56 supresiones, 36 sustituciones y 26 ampliaciones. 


\begin{tabular}{|c|c|c|}
\hline Estrategia & Ejemplos & Porcentaje \\
\hline Reescritura & 66 & $36 \%$ \\
\hline Supresión & 56 & $30 \%$ \\
\hline Sustitución & 36 & $20 \%$ \\
\hline Ampliación & 26 & $14 \%$ \\
\hline
\end{tabular}

Tabla 2. Cuantificación de estrategias en Los ojos de Ezequiel están abiertos

Según muestra la tabla 2, la reescritura fue, con el 36\% de los casos detectados, la estrategia más utilizada en la remodelación de la novela, en la que también se detectaron supresión (30\%), sustitución (20\%) y ampliación (14\%). Llama la atención el aumento de los casos de ampliación, algunos de los cuales son verdaderos ejercicios de creatividad que, motivados por fuertes consideraciones ideológicas, crean un poderoso discurso político, como el que aquí observamos:

\begin{tabular}{|l|l|}
\hline $\begin{array}{l}\text { TO } \\
\mathbf{( 1 9 4 9 )}\end{array}$ & $\begin{array}{l}\text {-Le marxisme est la seule théorie politique sérieuse, dit le moine. } \\
\text { C'est à la fois le moteur de l'histoire et son fossoyeur, la vérité de } \\
\text { Dieu vue par les yeux du Diable... }\end{array}$ \\
\hline $\begin{array}{l}\text { TM } \\
\mathbf{( 1 9 5 5 )}\end{array}$ & $\begin{array}{l}\text {-El marxismo es la única teoría política seria -dice el monje-. Es } \\
\text { a la vez motor de la historia y su sepulturero, la verdad de Dios } \\
\text { vista por los ojos del diablo, el Evangelio del Anticristo... }\end{array}$ \\
\hline
\end{tabular}

Tabla 3. Ejemplo de ampliación detectado en Los ojos de Ezequiel están abiertos

El que recoge la tabla 3 es uno de los numerosos casos que encontramos a lo largo de la traducción, en la que abundan ejemplos de ampliación de contenido del TO y con los que se pretende transmitir un mensaje ideológico en el TM. En este fragmento, se ha aprovechado las disquisiciones filosóficas e ideológicas del personaje para demonizar al marxismo, añadiendo elementos que no se encuentran en el TO. El marxismo no es solo el motor de la historia y su sepulturero, sino que además es "el evangelio del anticristo", una reflexión un tanto virulenta que sirve para potenciar, una vez más, el discurso favorable al régimen. Lo mismo sucede en el siguiente ejemplo, en el que se emplea la estrategia de sustitución. 


\begin{tabular}{|l|l|}
\hline $\begin{array}{l}\text { TO } \\
(\mathbf{1 9 4 9 )})\end{array}$ & $\begin{array}{l}\text { La mort d'une abeille assassinée par sa reine est chargée d'autant } \\
\text { de sens que les massacres de Dachau. }\end{array}$ \\
\hline TM & La muerte de una abeja asesinada por su reina está tan cargada de \\
$\mathbf{( 1 9 5 5 )}$ & tanta significación como las mortandades de Dachau. \\
\hline
\end{tabular}

Tabla 4. Ejemplo de sustitución detectado en Los ojos de Ezequiel están abiertos

La tabla 4 recoge un ejemplo de sustitución que, a través de una simple modificación, neutraliza el contenido censurable del TO. Al parecer, la referencia al horror del Holocausto podía traer recuerdos pocos favorables para el régimen franquista. Así tuvo que considerarlo el censor, que decidió suavizar el párrafo introduciendo una nueva traducción alternativa para el término massacres. En lugar de masacre, matanza o exterminio, se optó por mortandades, un término a todas luces más neutral y menos incómodo. Esta versión manipulada es la única que llegó a España de Abellio hasta que en 2011 Duomo Ediciones recuperara la traducción original, tal como Vila Selma la entregó a la editorial Escelicer. El estudio realizado, sin embargo, nos lleva a concluir que esta versión tampoco es íntegra y fiel, sino que presenta casos de la autocensura practicada por Selma, una práctica muy extendida entonces, como nos confirmaba Manuel Serrat Crespo:

Tuvimos que lanzarnos a especular con los límites de lo "permisible". Y en esta batalla, los editores solían mostrarse siempre -en mi recuerdomás timoratos porque no solo se jugaban el dinero de la posible "sanción administrativa" sino también todo lo invertido en la publicación "secuestrada".

En esta obra de Abellio, la censura está orientada a transformar importantes disquisiciones sobre religión, guerra y política en un discurso favorable al régimen, un ejercicio que se hizo en connivencia con el traductor y el editor de Escelicer. Esto demuestra hasta qué punto la cadena editorial tenía interiorizados los criterios censorios, bien por temor a represalias, bien por afinidad con los valores oficiales.

\footnotetext{
${ }^{3}$ Entrevista realizada a Manuel Serrat Crespo en el marco de la presente investigación.
} 


\subsection{La escritura invisible, de Arthur Koestler}

The Invisible Writing fue publicada en España en 1974 por Alianza en una edición de 199 páginas traducidas por Alberto Luis Bixio, que también se había encargado de la versión bonaerense de Emecé Editores veinte años atrás. Alianza presentó la obra a consulta el 21 de febrero de 1974. Todos los documentos referentes a este proceso administrativo quedan recogidos en el expediente 2308/74. El censor advierte ya en el primer informe que La escritura invisible constituye un libro "delicado" sobre los años que Koestler pasó en España durante la Guerra Civil. Pese a lo "inoportuno" que resultaría su publicación por una editorial española, existen razones implícitas que justifican una nueva versión de este libro: se tiene constancia de que circula de forma difundida una versión latinoamericana y, por lo tanto, limpia de censura franquista. El censor celebra que el autor se muestre "no resentido" y subraya el hecho de que la segunda parte del libro trate de "su desilusión y apartamiento del partido comunista". Este detalle tampoco escapa al segundo censor, quien ratifica la decisión del primero alegando: "el tratamiento de la segunda parte da a la obra cierto aspecto positivo por cuanto pone al descubierto la falsedad e hipocresía del comunismo" y autoriza la obra con tachaduras. Estas se centran en los ataques contra el régimen y Franco: "Los calificativos de fascista y dictador son frecuentes". De modo que La escritura invisible, que también pone de manifiesto los abusos del régimen y las atrocidades cometidas en la Guerra Civil, pasa a ser un texto que, con los retoques oportunos, puede resultar conveniente y hasta favorable. El análisis revela 12 ejemplos de censura: 6 supresiones, 5 reescrituras y 1 sustitución.

\begin{tabular}{|c|c|c|}
\hline Estrategia & Ejemplos & Porcentaje \\
\hline Supresión & 6 & $50 \%$ \\
\hline Reescritura & 5 & $41 \%$ \\
\hline Sustitución & 1 & $9 \%$ \\
\hline
\end{tabular}

Tabla 5. Cuantificación de estrategias censorias halladas en La escritura invisible

La tabla 5 indica que la censura recurrió mayoritariamente a la supresión con un $50 \%$ de los ejemplos. La escritura invisible sufrió únicamente doce alteraciones textuales que, no obstante, cumplieron su objetivo, esto es, reforzar el tono anticomunista y eliminar los ataques 
directos contra Franco y su régimen. Para apreciar el impacto de estas marcas, determinantes para el conjunto de la obra, pasamos a comentar un ejemplo de reescritura que, en este caso, representa el $41 \%$ de los ejemplos detectados.

\begin{tabular}{|l|l|}
\hline $\begin{array}{l}\text { TO } \\
\mathbf{( 1 9 5 4 )}\end{array}$ & $\begin{array}{l}\text { It was by no means abnormal for them, in the early 'thirties, to } \\
\text { regard Fascism as the main threat, and to be attracted, in varying } \\
\text { degrees, by the great social experiment in Russia. Even to-day, } \\
\text { about one quarter of the electorate in France and Spain, and } \\
\text { a much higher percentage among the intellectuals, regard it as } \\
\text { "normal" to vote for the Communist Party. }\end{array}$ \\
\hline $\begin{array}{l}\text { TM } \\
\mathbf{( 1 9 7 4 )}\end{array}$ & $\begin{array}{l}\text { En modo alguno era signo de anormalidad, en ellos, en los años } \\
\text { que siguieron a 1930, considerar el fascismo como la amenaza } \\
\text { principal y verse atraídos, en distintos grados, por el gran } \\
\text { experimento social que tenía del electorado de Francia e Italia, y } \\
\text { con un porcentaje aún mucho más elevado entre los intelectuales, } \\
\text { considera normal votar por el Partido Comunista. }\end{array}$ \\
\hline
\end{tabular}

Tabla 6. Ejemplo de reescritura detectado en el análisis de La escritura invisible

Este ejemplo de reescritura, un tipo de sustitución que se sirve del contenido censurable en el TO para introducir un cambio ideológico en el TM, se realiza a través de una concatenación de estrategias: se elimina, por un lado, contenido potencialmente peligroso (alusión favorable a Rusia) mientras que por otro se modifican algunos datos (el electorado español pasa a ser italiano; más revelador aún: la realidad temporal se altera también: even to-day queda fagocitado por la censura y las tendencias electorales procomunistas actuales pasan a ser de 1930). Koestler, que sufrió la revolución "traicionada" y la victoria del fascismo, quería alertar de los peligros de las ideologías totalitarias. Pero su visión llegó sesgada a los lectores españoles: ya no alertaba del totalitarismo, sino únicamente de la desilusión comunista. Alianza tuvo que acatar la decisión de la Administración; de otro modo, afirma Beatriz de Moura en su entrevista:

El editor podía cargar con las consecuencias tanto morales como materiales (en el mejor de los casos, contemplar, tragando bilis, cómo la policía destruía de madrugada en la imprenta toda la edición y, además, correr con todos los gastos consiguientes) ${ }^{4}$.

\footnotetext{
${ }^{4}$ Entrevista realizada a Beatriz de Moura en el marco de la presente investigación.
} 
Doce alteraciones quizá no sean muchas, pero su efecto fue mordaz; la censura consiguió su propósito: crear un discurso anticomunista, favorable a los intereses del régimen.

\section{Análisis comparativo}

Una vez analizadas individualmente cada una de las obras y realizada una valoración de los resultados, en este apartado se realiza un análisis comparativo de las tres obras en conjunto para tratar de desentrañar el comportamiento censor que se esconde tras ellas.

\begin{tabular}{|l|c|c|c|c|c|}
\hline \multicolumn{1}{|c|}{ Obra } & Supr. & Reesc. & Sust. & Ampl. & Total marcas \\
\hline 1984 & 42 & 8 & 2 & 1 & 53 \\
\hline Ezequiel & 56 & 66 & 36 & 26 & 184 \\
\hline Escritura & 6 & 5 & 1 & 0 & 12 \\
\hline Porcentaje & $\mathbf{4 2 \%}$ & $\mathbf{3 2 \%}$ & $\mathbf{1 5 \%}$ & $\mathbf{1 1 \%}$ & $\mathbf{1 0 0 \%}$ \\
\hline
\end{tabular}

Tabla 7. Número de marcas y porcentaje de estrategias censorias halladas en las obras del corpus

Esta tabla recapitulativa muestra que la supresión es la estrategia más utilizada por la censura, con el $42 \%$ de los casos, mientras que en el $58 \%$ de los casos se observa una tendencia a manipular el contenido censurable del TO ya sea sustituyéndolo por otro más neutral $(15 \%)$, ampliándolo (11\%) o reescribiéndolo (32\%) para conseguir un cambio ideológico. Es lo que observamos en 1984, donde el Estado totalitario ideado por Orwell se transforma en un infierno comunista, como pretendía la Administración. También Koestler, testigo de la Guerra Civil española y de los primeros años del franquismo, vio manipulado su testimonio en La escritura invisible, en el que se eliminó todo tono acusatorio hacia el régimen. En el caso de Los ojos de Ezequiel están abiertos, la censura transformó importantes disquisiciones sobre religión, guerra y política en un discurso simpatizante con la Iglesia; un ejercicio que se hizo en connivencia con el traductor y el editor de Escelicer. La función de este tipo de censura detectado en obras con contenido político subversivo no es omitir sino reescribir, introduciendo elementos ajenos al TO. Se trata de una "metacensura" que, más allá de los recursos tradicionales, transforma el mensaje original de tal modo que pueda 
ser utilizado para beneficiar al régimen, para emplearlo como refuerzo de la ortodoxia, los valores y la doctrina que constituyen la arquitectura ideológica construida por este y que sitúa a la traducción como una herramienta de control ideológico, como un recurso propagandístico que da a la censura una dimensión más amplia y poderosa.

\section{Conclusiones}

Este trabajo buscaba ahondar en esta otra faceta de la traducción, menos idealizada, que actúa como herramienta propagandística al servicio del poder. La metodología adoptada, una propuesta mixta que combina el análisis cuantitativo y cualitativo de los datos, nos ha permitido establecer un patrón de comportamiento para estas obras. La incorporación de material extratextual nos ha proporcionado además una visión más amplia y cercana de la realidad franquista y de los motivos que impulsaron la censura de las obras. Los resultados obtenidos en esta investigación pueden, de este modo, resumirse en dos conclusiones principales. En primer lugar, se observa una tendencia a modelar con fines propagandísticos, con un 58\% de casos de reescritura, sustitución y ampliación, orientados a transformar el contenido subversivo del TO. En segundo lugar, cada uno de los textos encierra pistas interesantes sobre los motivos que impulsaron la censura de sus páginas y sobre el efecto que se deriva de ella: La escritura invisible, de Koestler, acabó transformándose en un discurso favorable a la causa de Franco; 1984, de Orwell, en un discurso anticomunista; Los ojos de Ezequiel están abiertos, en un discurso simpatizante con la Iglesia. Este estudio revela un uso frecuente de "metacensura", una estrategia que se vale de los recursos tradicionales y va más allá, reescribiendo con fines propagandísticos, un tipo de censura que ofrece multitud de posibilidades para los investigadores que quieran ahondar en esta peculiar faceta de la traducción.

\section{Bibliografía}

Abellán, Manuel. 1980. Censura y creación literaria en España (1939-1976). Barcelona: Península.

Abellán, Manuel. 1982. Censura y autocensura en la producción literaria española. Nuevo Hispanismo 1: 169-180.

Abellio, Raymond. 1949. Les Yeux d'Ezéchiel sont ouverts. París: Gallimard. 
Abellio, Raymond. 1955. Los ojos de Ezequiel están abiertos. Madrid: Escelicer. [Traducción de José Vila Selma].

Cisquella, Georgina; Erviti, José Luis \& Sorolla, José A. 1977. Diez años de represión cultural: la censura de libros durante la ley de prensa (19661976). Barcelona: Anagrama.

González Ruiz, Victor. 2005. Traducción e ideología en el ámbito de los medios audiovisuales: el caso de los títulos cinematográficos. En Chaume Varela, Frederic; Santamaría Guinot, Laura \& Zabalbeascoa Terran, Patrick (ed.) La traducción audiovisual: investigación, enseñanza y profesión. Granada: Comares, 127-138.

Koestler, Arthur. 1954. The Invisible Writing. Londres: Collins \& Hamish Hamilton.

Koestler, Arthur. 1974. La escritura invisible. Madrid: Alianza. [Traducción de Alberto Luis Bixio].

Laprade, Douglas Edward. 2006. Censura y recepción de Hemingway en España. Valencia: Publicacions de la Universitat de València.

Lázaro, Alberto. 2002. La sátira de George Orwell ante la censura española. En Falcés Sierra, Marta; Díaz Dueñas, Mercedes \& Pérez Fernández, Jose María (ed.) Actas de la 25. ${ }^{a}$ Conferencia Internacional de AEDEAN. Granada: Universidad de Granada, 1-15.

Lázaro, Alberto. 2004. H. G. Wells en España: estudio de los expedientes de censura (1939-1978). Madrid: Verbum.

Meseguer, Purificación. 2015. Traducción y reescritura ideológica bajo el franquismo: La falta del abate Mouret, de Émile Zola. Çedille: revista de estudios franceses 11: 389-412.

Merino, Raquel. 2007. Traducción y censura en España (1939-1985). Estudios sobre corpus de cine, narrativa y teatro. Vitoria y León: UPV/EHU y ULE.

Orwell, George. 1949. Nineteen Eighty Four. Londres: Secker \& Warburg.

Orwell, George. 1952. 1984. Madrid: Destino. [Traducción de Rafael Vázquez Zamora].

Rabadán, Rosa. 2000. Traducción y censura inglés-español: 1939-1985. Estudio preliminar. León: Universidad de León.

Ruiz Bautista, Eduardo. 2008. Tiempo de censura: La represión editorial durante el franquismo. Gijón: Trea.

Santoyo, Julio César. 2000. Traducción y censura: Mirada retrospectiva a una historia interminable. En Rabadán, Rosa (ed.) Traducción y censura inglés-español: 1939-1985. Estudio preliminar. León: Universidad de León, 291-309.

Savater, Fernando. 1996. Ángeles decapitados. La desertización cultural bajo el franquismo. Claves de la Razón Práctica 59: 8-13. 